\title{
The power of positive deviance
}

\author{
David R Marsh, Dirk G Schroeder, Kirk A Dearden, Jerry Sternin, Monique Sternin
}

Identifying individuals with better outcome than their peers (positive deviance) and enabling communities to adopt the behaviours that explain the improved outcome are powerful methods of producing change

The most efficient way to improve health is to use locally available, sustainable, and effective approaches. In the 1970 s policy developers tested the concept that public health interventions could be designed around uncommon, beneficial health behaviours that some community members already practised. $^{12}$ This concept-known as positive deviance ${ }^{3}{ }^{4}$ - was used successfully to improve the nutritional status of children in several settings in the1990s. . $^{5-10}$ Recently, the approach has also been applied to newborn care, child nutrition, rates of contraception, safe sexual practices, and educational outcomes. ${ }^{11-13}$ In this article we describe how the approach works, the evidence that it is effective, and possible future applications.

\section{How does positive deviance work?}

Positive deviant behaviour (box) is an uncommon practice that confers advantage to the people who practise it compared with the rest of the community. Such behaviours are likely to be affordable, acceptable, and sustainable because they are already practised by at risk people, they do not conflict with local culture, and they work..$^{15}$ For example, in Egypt, contrary to custom, parents of poor but well nourished children were found to feed their children a diet that included eggs, beans, and green vegetables. Child nutrition programmes that provided opportunities to parents of malnourished children to follow this and other new behaviours, such as hand washing and hygienic food preparation, improved child growth (figure).

The positive deviance approach involves partnering with communities to:

- Develop case definitions

- Identify four to six people who have achieved an unexpected good outcome despite high risk

- Interview and observe these people to discover uncommon behaviours or enabling factors that could explain the good outcome

- Analyse the findings to confirm that the behaviours are uncommon and accessible to those who need to adopt them

- Design behaviour change activities to encourage community adoption of the new behaviours

- Monitor implementation and evaluate the results.

This approach to behavioural and social change takes advantage of the community's existing assets or

\section{Positive deviance}

Positive deviance is the observation that in most settings a few at risk individuals follow uncommon, beneficial practices and consequently experience better outcomes than their neighbours who share similar risks. ${ }^{14}$

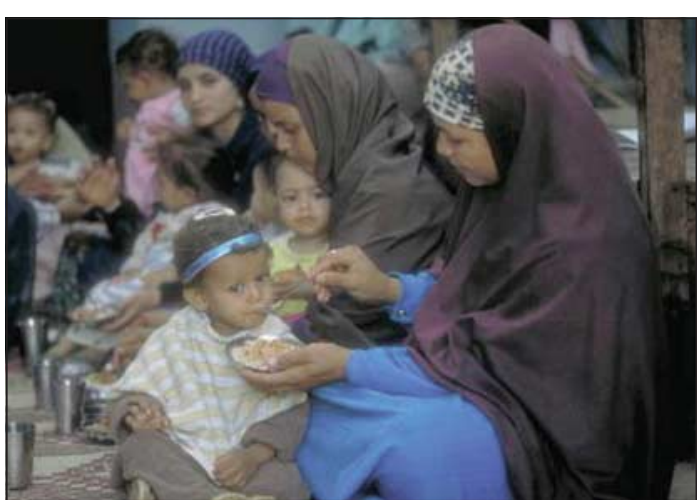

Mother learning new feeding practices at a mother-infant group learning centre in Abo Sidom, Egypt

strengths. In contrast, most international health initiatives are prescriptive, top down, or donor driven and difficult to sustain without ongoing external resources.

The approach facilitates three important processes: social mobilisation, information gathering to craft interventions, and behaviour change.

Social mobilisation-We have found that, even during the initial explanation of the positive deviance concept in communities, local villagers respond with excitement and enthusiasm to get started. Participants have said that they are motivated by learning that they are doing something right and that a successful solution to their problem already exists within their community, instead of receiving criticism for their inadequacies.

Information gathering-In-depth inquiries, community norms studies, and community vetting are used to identify the transferable behaviours and enabling factors that are likely to account for the good outcome of the identified individual or family (see table A on bmj.com). Depending on the project aim, policy developers seek behaviours that explain good health status outcomes (for example, good weight for age) or enablers and other behaviours that explain good behavioural outcomes (such as protecting daughters from girl trafficking).

Behaviour change-Some behaviours are so affordable and accessible (such as a clean blade to cut a newborn baby's cord) that enthusiasm for immediate adoption ripples through the community, even before the formal intervention starts. Other behaviours change more slowly through interventions that encourage new behaviours.

\section{Experience and evidence from the field}

Through a series of increasingly rigorous studies, Save the Children has evaluated the effectiveness of the

Further examples of the use of positive deviance and references w1-w13 are on bmj.com
Save the Children Federation (USA), Westport,

Connecticut, USA David R Marsh senior child survival advisor

Rollins School of Public Health,

Emory University,

Atlanta, Georgia,

USA

Dirk G Schroeder associate professor

Brigham Young University, Provo, Utah, USA

Kirk A Dearden associate professor

Positive Deviance Initiative, Tufts University, Medford, Massachusetts, USA Jerry Sternin director

Cambridge, Massachusetts, USA Monique Sternin independent consultant Correspondence to: D R Marsh, 31 Wildflower Drive, Amherst, Massachusetts, USA dmarsh@ savechildren.org

BMJ 2004;329:1177-9 
positive deviance approach to reduce childhood malnutrition in Vietnam (table B on bmj.com). In the early 1990 s, Sternin et al showed a $74 \%$ reduction in severe malnutrition among children younger than 3 years old who participated in positive deviance programmes. ${ }^{56}$ Mackintosh et al revisited some of these children in 1998, three years after the intervention stopped..$^{16}$ They found that these children, and especially their younger siblings born after completion of the programme, had better nutritional status than age and sex matched children who did not live in intervention communities. Semistructured interviews with mothers strongly suggested that the superior nutritional status of the younger siblings was due to better care from a younger age-improved practices that their mothers had learnt during the programme.

The strongly positive and consistent results from these programmatic evaluations suggested the approach was successful, but their relatively weak study designs limited our ability to attribute causality to the interventions. We therefore conducted a large, randomised, prospective trial of the approach in 12 communes in northern Vietnam. ${ }^{17}$ Save the Children conducted monthly measurements on 240 malnourished children (120 in the intervention and nonintervention communes) for six months and then at 12 months. Compared with children in randomly selected non-intervention communes, the intervention children grew better, ${ }^{18}$ ate and breastfed more often, ate larger portions, consumed more energy, ${ }^{17}$ experienced less respiratory infection, ${ }^{19}$ and had mothers who were more likely to confidently share new knowledge about child care and feeding with their neighbours. ${ }^{20}$ Many effects, such as improved diet and decreased morbidity, occurred among all children, even those not sufficiently malnourished to attend the group learning activities. This finding is consistent with behaviour change through mothers sharing new behaviours with one another.

Use of the positive deviance approach outside nutrition and child survival is limited but growing. It has been used to promote condom use among commercial sex workers in Georgia and Indonesia, improve family planning methods in Guatemala, and improve outcome of pregnancy in Egypt. ${ }^{13}{ }^{21}$ It has also been used in efforts to improve educational outcomes in the United States ${ }^{\mathrm{w1}}$ to develop a "best practices" tool in Argentina, ${ }^{\text {w2 }}$ and in a university donor partnership to identify factors influencing sexual practices in West Africa. ${ }^{\text {w3 }}$ The Centre for Development and Population Activities used the positive deviance approach to advocate against female genital cutting in Egypt at the community level and nationally through television using the actual words of the positive deviance individuals, ${ }^{\mathrm{w} 4}$ and the Government of Egypt will apply the approach in 60 villages. The US healthcare and business communities are beginning to recognise the power of positive deviance. ${ }^{\text {w5-w8 }}$

\section{Advantages and disadvantages}

The success of the approach rests on its ability to mobilise the community to identify role models within its midst who use uncommon, but demonstrably successful, strategies to tackle common problems. Use

\section{Summary points}

Even in the poorest communities, a few individuals or families achieve good health

Positive deviance is a quick, low cost method to identify the strategies used by these people and encourage the rest of the community to adopt them

The approach has been used successfully, mainly to improve child health

The potential for the approach to help communities to gain better health or other social benefits is vast and largely untapped

of this approach has wider benefits. Firstly, it serves equity, in that it is informed by the wisdom of disadvantaged "doers" of healthy behaviours and provides solutions accessible to those with similar socioeconomic constraints. ${ }^{\mathrm{w} 9}$ Secondly, it introduces a generic approach for local problem solving. Indeed, intervention communities commonly launch parallel activities, such as cost sharing for local road construction or successfully demanding social services. Thirdly, positive deviance enhances local research capacity for controlling disease in relevant, affordable, sustainable ways. Lastly, and perhaps most importantly, the approach reveals at least partial solutions today to challenges rather than waiting for long term development. Clearly planners must address the complex underlying development challenges, but the approach permits some action now, which builds trust and enthusiasm for the long haul.

The approach does, however, have limitations. It requires discovering uncommon positive examples, typically at a prevalence of $1-10 \%$. Rare examples are costly to identify, and common examples fail to stimulate new thinking. By extension, the approach is inappropriate for settings where positive behaviour is impossible due to non-availability of relevant services or foods. Scale-up requires many people with skills in community mobilisation, participatory research, and positive deviance, which may limit uptake. On the other hand, training materials, ${ }^{\text {w10-w12 }}$ workshops, and technical assistance are increasingly available.

Some people have questioned the efficiency of the approach, given the presumed limited generalisability of findings from local inquiries and the desire to mobilise each community through self discovery. Practitioners now need to test the assumption that positive deviance is, of necessity, a small scale approach by evaluating the effectiveness of different intensities of inquiry (number per population size). Similarly we should test reformulated approaches, such as a mass media advocacy campaign featuring testimonials by individuals with positive deviant behaviour. ${ }^{\mathrm{w} 13}$ Likewise, we should systematically describe and value the additional unintended benefits accrued by communities that have taken part in positive deviance programmes. 


\section{Future challenges?}

The potential role for positive deviance is vast. For example, which rural Kenyan families optimally use insecticide impregnated bednets, and how can they motivate their neighbours? How can South African policy makers integrate the behaviours and thinking of teenagers who practise "safe sex"? What can we learn from a poor, uninsured Latina mother who succeeds in properly managing her child's diabetes or asthma? What about other intractable, deadly impasses of our time- the Kashmir crisis, Israeli-Palestinian mayhem, or insurgency in Iraq? We believe that positive deviance is a valuable tool that should be part of international health policy makers' toolbox for the 21st century.

Contributors and sources: DRM drafted the paper with input from all authors. JS and MS have designed and implemented positive deviance informed projects; DRM, DGS, and KAD have evaluated such projects in many countries. The information in the paper comes from publications and the authors' experiences.

Competing interest: None declared.

1 Wray JD. Can we learn from successful mothers? I Trop Pediatr Environ Child Health 1972;18:27.

2 Wishik SM, Van der Vynkt S. The use of nutritional 'positive deviants' to identify approaches for modification of dietary practices. Am J Pub Health identify approach

3 Zeitlin M, Ghassemi H, Mansour M. Positive deviance in child nutrition-with emphasis on psychosocial and behavioral aspects and implications for development. Tokyo: United Nations University, 1990.

Shekar M, Habicht J-P, Latham M. Positive-negative deviant analyses to improve programme targeting and services: example from Tamil Nadu Integrated Nutrition Project. Int J Epidemiol 1992;21:707-13.

5 Sternin M, Sternin J, Marsh D. Rapid, sustained childhood malnutrition alleviation through a "positive deviance" approach in rural Vietnam: preliminary findings. In: Keeley E Burkhalter BR, Wollinka O, Bashir N, eds. The hea th mutition molel: applications in Haiti, Viemam, and Bang The hearth nutrition model: applications in Haiti, Vietnam, and Bangladesh, Report of a Technical Meeting at World Relief Corporation, Wheaton, IL, June BASICS, 1997.

6 Sternin M, Sternin J, Marsh D. Scaling up a poverty alleviation and nutrition program in Viet Nam. In: Marchione T. Scaling up, scaling down: capacities for overcoming malnutrition in developing countries. Amsterdam: Gordon and Breach, 1999.

7 Bolles K, Speraw C, Berggren G, Lafontant JG. Ti Foyer (hearth) community-based nutrition activities informed by the positive deviance approach in Leogane, Haiti: A programmatic description. Food Nutr Bull 2002;23 (suppl 4):11-17

8 Food for the Hungry International. FY2001 annual report. http:// gme.fhi.net/fse/R2/docs/ISA\%20FY\%202001\%20Report.doc2004 (accessed 6 Oct 2004).

9 EcoYoff. Positive deviance-take 2. Living and learning newsletter 2003 Sep 21. http://ifnc.tufts.edu/pdf/ecoyoff21.pdf (accessed 11 Oct 2004).

10 Sethi V, Kashyap S, Seth V, Agarwal S. Encouraging appropriate infant feeding practices in slums: a positive deviance approach. Pakistan J Nutr 2003;2:164-6.

11 Dearden K, Quan N, Do M, Marsh DR, Schroeder G, Pachón H, et al. What influences health behavior? Learning from caregivers of young children in Vietnam, Food Nutr Bull 2002;23(suppl 4):119-29.

12 Marsh DR, Sternin M, Khadduri R, Ihsan T, Nazir R, Bari A, et al. Identification of model newborn care practices through a positive deviance inquiry to guide behavior change interventions in Haripur, Pakistan. Food inquiry to guide behavior change intr
Nutr Bull 2002;23(suppl 4):109-18.

13 Ahrari M, Kuttab A, Khamis S, Farahat AA, Darmstadt GL, Marsh DR, et al. Socioeconomic and behavioral factors associated with successful pregnancy outcomes in upper Egypt: a positive deviance inquiry. Food Nutr Bull 2002;23:83-8.

14 Berggren WL, Wray JD. Positive deviant behavior and nutrition education. Food Nutr Bull 2002;23(suppl 4):9-10.

15 Marsh DR, Schroeder DG, The positive deviance approach to improve health outcomes: experience and evidence from the field: preface. Food Nutr Bull 2002;23( suppl 4):5-8

16 Mackintosh AT, Marsh DR, Schroeder DG, Sustainable positive deviant child care practices and their effects on child growth in Viet Nam. Food Nutr Bull 2002;23( suppl 4):18-27.

17 Marsh DR, Pachón H, Schroeder DG, Ha TT, Dearden K, Lang TT, et al. Design of a prospective, randomized evaluation of an integrated nutrition program in rural Viet Nam. Food Nutr Bull 2002;23(suppl 4):36-47.

18 Schroeder DG, Marsh DR, Ding B, Pachón H, Ha TT, Dearden KD, et al. Impact of an intervention on Vietnamese children's growth. Food Nutr Bull 2002;23(suppl 4):53-61.

19 Sripaipan T, Schroeder D, Marsh DR, Pachón H, Dearden K, Ha TT, et al. Do community-based nutrition programs reduce morbidity? A case from Vietnam. Food Nutr Bull 2002;23(suppl 4):70-7.

20 Hendrickson JL, Dearden KA, Pachon H, An NH, Schroeder DG, Marsh DR. Empowerment in rural Viet Nam: Exploring changes in mothers and health volunteers in the context of an integrated nutrition project, Food Nutr Bull 2002;23(suppl 4):86-94.

21 Positive Deviance Initiative. Projects. www.positivedeviance.org/projects (accessed 11 Oct 2004)

(Accepted 11 August 2004)

\title{
Kangaroo Mother Care, an example to follow from developing countries
}

\author{
Juan Gabriel Ruiz-Peláez, Nathalie Charpak, Luis Gabriel Cuervo
}

Caring for low birthweight infants imposes a heavy burden on poor countries. An effective healthcare technique developed in 1978 may offer a solution to this problem and additionally be of use in wealthy countries too

\section{Introduction}

Each year about 20 million infants of low birth weight are born worldwide, which imposes a heavy burden on healthcare and social systems in developing countries. ${ }^{1}{ }^{\text {w1 }}$ Medical care of low birthweight infants is complex, demands an expensive infrastructure and highly skilled staff, and is often a very disruptive experi-

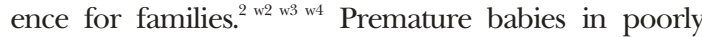
resourced settings often end up in understaffed and ill equipped neonatal care units, that may be turned into potentially deadly traps by a range of factors colludingfor example, malfunctioning incubators, broken monitors, overcrowding, nosocomial infections, etc.

In 1978 Edgar Rey, a Colombian paediatrician concerned with the problems arising from a shortage of incubators and the impact of separating women from newborns in neonatal care units, developed Kangaroo Mother Care (KMC), ${ }^{3}$ a healthcare technique for low birthweight infants that is at least as effective as traditional care in a neonatal care unit. ${ }^{45}$

\section{What does KMC entail?}

In KMC, babies weighing $2000 \mathrm{~g}$ or less at birth and unable to regulate their body temperature remain with their mothers as incubators, main source of stimulation, and feeding. Newborns are attached to mothers
Clinical

Epidemiology and Biostatistics Unit, l of Medicine, Javeriana University, Bogotá, Colombia Juan Gabriel

Ruiz-Peláez professor continued over

BMJ 2004;329:1179-82 